\title{
Efficacy and Treatment Strategies in Advanced Cancers with Liver Metastasis Receiving Atezolizumab Therapy
}

\author{
Wen-Jing Yin ${ }^{1,2, *}$ \\ Si-Cong $\mathrm{Ma}^{3, *}$ \\ Zhong-Yi Dong (iD) ${ }^{3, *}$ \\ Meng $X u^{\prime}$ \\ Wu Mao ${ }^{4}$ \\ 'Department of Oncology, The First \\ Affiliated Hospital of Jinan University, \\ Guangzhou, People's Republic of China; \\ ${ }^{2}$ Department of Radiation Oncology, \\ Affiliated Cancer Hospital \& Institute of \\ Guangzhou Medical University, \\ Guangzhou, People's Republic of China; \\ ${ }^{3}$ Department of Radiation Oncology, \\ Nanfang Hospital, Southern Medical \\ University, Guangzhou, People's Republic \\ of China; ${ }^{4}$ Department of Surgery, \\ Guangdong Women and Children \\ Hospital, Guangzhou, People's Republic \\ of China
}

*These authors contributed equally to this work
Background: Atezolizumab has been used to treat patients with liver metastasis (LM). However, whether atezolizumab is superior to standard of care therapy in an all-comer or selective population with LM is still uncertain.

Methods: A pooled analysis based on 10 randomized controlled trials was conducted to evaluate the clinical benefit of atezolizumab versus standard therapy in patients stratified by liver metastatic status, followed by biomarker-based individual analyses of the non-small cell lung cancer (NSCLC) cohort (OAK and POPLAR studies) and urothelial cancer cohort (IMvigor210 study).

Results: The pooled analysis demonstrated an overall survival (OS) improvement using atezolizumab treatment versus standard therapy across cancer types and treatment lines regardless of liver metastatic status. However, the efficacy of atezolizumab in patients with LM from the second-line setting was limited, based on the individual analysis of NSCLC cohorts $(P=0.053)$. PD-L1 strong expression emerged as a predominant biomarker $(P=$ 0.015 ) to screen atezolizumab-advantageous patients with LM. Notably, the combination of PD-L1 and LM improved the predictive power for atezolizumab therapy in both NSCLC and urothelial cancer cohorts. Exploratory translational analysis revealed that strong expression of PD-L1 might have reversed the non-inflamed immune phenotype of liver metastasis, thus sensitizing these patients to immunotherapy.

Conclusion: Our study demonstrated a preferable efficacy of atezolizumab in patients with LM as first-line therapy over standard of care therapy, while sensitive patients should be selected in second-line settings. PD-L1 was demonstrated as the most effective biomarker for screening atezolizumab-advantageous patients with LM.

Keywords: liver metastasis, atezolizumab, PD-L1, pooled analysis, advantageous

\section{Background}

The advent of immune checkpoint blockade (ICB) therapy has brought about a revolution and altered the treatment paradigm for patients with advanced or metastatic cancers. ${ }^{1-4}$ However powerful, multiple questions remain unanswered in this area. Getting an insight into organ-specific tumor-immune interactions turns out to be one of the top challenges, ${ }^{5}$ since the organ in which a tumor resides is a vital component of those factors relevant to an anti-tumor immune response. ${ }^{6}$ Divergent responses emerge among tumors metastasized to different sites. ${ }^{2}$ Therefore, further studies are warranted to elucidate the immunotherapy response of specific metastatic sites in cancer immunology. 
The liver, with a distinctive organ-specific immunity, is one of the most common sites of visceral metastasis for a variety of cancers, including lung, colon, and head and neck cancers. ${ }^{7,8}$ The presence of baseline liver metastasis (LM) is associated with treacherous prognosis. One recent study, which included over 20,000 patients, reported that those with lung cancer metastasized to the liver had an OS of 3 months. ${ }^{9}$ Being refractory to treatment and showing poor responses, conventional therapies failed to improve their prognosis. ${ }^{10-12}$ In addition, in some patients with LM, unacceptable toxicity from chemotherapy was associated to hepatic insufficiency. Thus, ICB therapy might open a new avenue for them. Notably, liver tissue harbors a distinct immune microenvironment, with myeloidderived suppressor cells, Kupffer cells, and liver dendritic cells driving an immunosuppressive network, thwarting the infiltration and activation of $\mathrm{CD} 8^{+}$effector $\mathrm{T}$ cells, ${ }^{13}$ which may thereby influence responses to immunotherapy in liver metastasis. ${ }^{14} \mathrm{~A}$ recently updated analysis report regarding non-small cell lung cancer (NSCLC) patients participating in CheckMate 017 and CheckMate 057 trials demonstrated long-term clinical benefits from nivolumab in the liver metastatic population; ${ }^{11}$ however, its superiority remains controversial, since some other studies failed to confirm a significant benefit. ${ }^{15}$ Disputes also exist in patients across different treatment lines (firstor second-line settings). Additionally, findings between the IMmotion151 and IMpassion130 studies showed inconsistency among various types of cancer, ${ }^{16,17}$ indicating that researches on ICB responses in LM have not achieved an intact and authoritative theory. Therefore, further exploration of treatment paradigms for patients with LM is crucial for tailored and precision therapy as well as for improving prognosis.

Regarding paradoxical results from previous studies, we investigated the efficacy of the ICB response in the setting of LM, among various cancer types, in different treatment modes. Atezolizumab, as a promising antiprogrammed death ligand 1 (anti-PD-L1) monoclonal antibody, has been widely evaluated in multiple randomized controlled trials, during which a large amount of data from liver metastatic patients were generated, making it possible for us to conduct this study comparing immunotherapy (especially atezolizumab) with standard therapy comprehensively across regimens and cancer types. Moreover, though LM is characterized by induction of immune escape through various mechanisms, ${ }^{13,18}$ some patients afflicted by LM can still benefit from ICB therapy, implying that there exist subgroups of patients with different responses to atezolizumab. This information prompted us to explore potential biomarkers for the characterization of atezolizumab-advantageous populations.

Thus, we set out an integrated study of a pooled population with LM based on 10 randomized controlled trials of atezolizumab versus standard of care therapy, followed by an individual analysis in the POPLAR (phase II) and OAK (phase III) trials. Atezolizumab-advantageous biomarkers were also screened in the OAK cohort and further validated in a urothelial carcinoma (UC) cohort collected from the Phase II IMvigor210 trial. Finally, the underlying mechanisms were analyzed based on changes in the immune profiles.

\section{Methods}

\section{Clinical Cohorts}

\section{Pooled Analysis}

A total of 7028 patients from 10 randomized controlled trials evaluating the efficacy of atezolizumab versus standard of care therapy were pooled across studies, including 4960 patients from 7 first-line trials and 2068 patients from 3 second-line trials. Of these, there were 1486 patients (21.1\%) with LM and 5542 patients (78.9\%) without LM at baseline. Basic information of the corresponding randomized controlled trials is summarized in Table S1, and the detailed searching strategy and selection criteria of the enrolled studies are provided in the Supplementary Methods.

\section{Individual Analysis}

Two NSCLC cohorts were collected from POPLAR and OAK, both of which were randomized controlled trials comparing atezolizumab and docetaxel (the standard chemotherapy) in second-line patients who progressed on previous cytotoxic chemotherapy. ${ }^{19,20}$ We also collected a UC cohort from IMvigor210, a single-arm trial evaluating the efficacy of atezolizumab as a second-line regimen. ${ }^{2}$ Baseline PD-L1 expression data of patients in OAK and IMvigor210 were evaluated using the SP142 immunohistochemistry assay, and scored as the percentage of PD-L1 expression in tumor cells (TC0: $<1 \%$, TC1: $\geq 1 \%$ and $<5 \%, \mathrm{TC} 2: \geq 5 \%$ and $<50 \%$, and TC3: $\geq 50 \%$ ) and immune cells (IC0: $<1 \%$, IC1: $\geq 1 \%$ and $<5 \%$, IC2: $\geq 5 \%$ and $<10 \%$, and IC $3: \geq 10 \%$ ). ${ }^{2,20}$

\section{Study Design}

This study was composed of two main stages. The objective of the first stage was to assess the efficacy of atezolizumab versus standard therapy with respect to baseline 
LM. In this stage, a pooled analysis of various cancers across treatment lines was conducted, followed by an individual analysis of a NSCLC cohort, combining OAK and POPLAR, to confirm the findings. The objective of the second stage was to establish a biomarker for risk stratification of liver metastatic patients who received atezolizumab. In this stage, the OAK cohort was used for biomarker screening and the IMvigor210 cohort for further validation (Figure S1). An exploratory translational analysis was subsequently performed to elucidate the underlying mechanism from the perspective of immune profiles. The primary outcome in this study was overall survival (OS), defined as the time lapse from the start of treatment to the date of death or the last follow-up. Patients included in clinical trials provided signed informed consent in accordance with their clinical study protocols. This study was approved by the Institutional Ethical Review Boards of the Affiliated Cancer Hospital \& Institute of Guangzhou Medical University (No.2021-001).

\section{Analysis of Transcriptomic Data}

Transcriptomic data from IMvigor210 were profiled with RNA sequencing (RNA-seq) and normalized using the transcripts per million (TPM) method. The activity of $\mathrm{CD} 8^{+} \mathrm{T}$ cells was quantified using the gene expression level of an eight-gene $\mathrm{T}$ cell effector signature, ${ }^{2,21}$ consisting of TBX21, GZMA, GZMB, IFNG, PRF1, CD8A, CXCL9, and CXCL10. Immune scores of various types of infiltrated immune cells were evaluated as the average expression level of genes composing each signature, which were determined in previous studies. ${ }^{22,23}$

\section{Statistical Analysis}

The pooled analysis of atezolizumab versus standard therapy with various cancers across treatment lines was performed using Review Manager version 5.3 (RevMan, Cochrane Collaboration, Oxford, England); subtotal and total hazard ratios (HRs) with $95 \%$ confidence intervals (CIs) were calculated in populations with and without LM. The Kaplan-Meier method was applied to compare OS between subgroups, and survival curves were drawn using GraphPad Prism (version 8.0.1). We estimated the HRs of atezolizumab versus docetaxel with $95 \%$ CIs and their corresponding $P$ values by $\log$ rank test in the OAK cohort to screen for potential biomarkers, using the survival package in $\mathrm{R}$ (version 3.6.1). The expression level of the eight-gene $\mathrm{T}$ cell effector signature and immune cell scores were compared across subgroups using the
Kruskal-Wallis test included in the ggpubr package in R. All $P$ values were based on two-tailed test and $P \leq$ 0.05 was considered to meet the conventional level of statistical significance.

\section{Results \\ Efficacy of Atezolizumab versus Standard Therapy in Terms of Baseline Liver Metastasis (LM)}

To investigate the influence of baseline liver metastatic status on the efficacy of atezolizumab, we first conducted a pooled analysis of atezolizumab versus standard of care treatment (chemotherapy or targeted therapy) based on 10 randomized controlled trials.

Progression-free survival (PFS) was significantly improved in patients receiving atezolizumab compared with those receiving a standard therapy, regardless of baseline liver metastatic status (non-LM: total HR 0.73, 95\%: CI $0.68-0.77$; LM: total HR 0.80 , 95\% CI: $0.71-0.91$ ) (Figure 1A). If treatment lines were taken into account, PFS was generally similar between treatments in the second-line setting, but a significant benefit from atezolizumab treatment was observed in the first-line setting, irrespective of the presence or absence of LM (non-LM: subtotal HR 0.68, 95\% CI: 0.64-0.74; LM: subtotal HR 0.74, 95\% CI: 0.64-0.84) (Figure 1A).

OS consistently favored atezolizumab over standard therapy in the liver metastatic subgroup (total HR 0.78 , 95\% CI 0.71-0.85) and the non-liver metastatic subgroup (total HR 0.78, 95\% CI: 0.69-0.89) (Figure 1B). Specifically, atezolizumab demonstrated an OS benefit versus standard therapy regardless of the first-line combination therapy (non-LM: subtotal HR $0.80,95 \%$ CI: 0. 70-0.90; LM: subtotal HR 0.77, 95\% CI: 0.64-0.92) or second-line monotherapy (non-LM: subtotal HR 0.76, 95\% CI: 0.67-0.86; LM: subtotal HR 0.80, 95\% CI: 0.66-0.98) (Figure 1B).

In addition to the pooled analysis, we conducted an individual analysis specifically in a second-line NSCLC cohort composed of trial data from POPLAR and OAK, to confirm the findings mentioned above. The Kaplan-Meier survival curve showed a predominant survival advantage of atezolizumab over docetaxel according to OS (median OS [mOS] 15.47 vs 10.94 months, $P<0.0001)$ in the nonliver metastatic population (Figure 1C). Nevertheless, as for the liver metastatic population, although we saw some evidence of improved OS in patients treated with 
A

\begin{tabular}{lc} 
& \multicolumn{1}{c}{ Non-liv } \\
\cline { 2 - 2 } Study or Subgroup & \multicolumn{1}{c}{$\begin{array}{l}\text { Hazard Ratio } \\
\text { IV, Fixed, 95\% CI }\end{array}$} \\
\hline Combination therapy & (first line) \\
IMmotion151 & $0.89[0.73,1.09]$ \\
IMpassion130 & $0.79[0.66,0.95]$ \\
IMpower130 & $0.59[0.49,0.71]$ \\
IMpower131 & $0.68[0.56,0.83]$ \\
IMpower132 & $0.56[0.46,0.68]$ \\
IMpower133 & $0.72[0.55,0.94]$ \\
IMpower150 & $0.64[0.54,0.76]$ \\
Subtotal (95\% Cl) & $\mathbf{0 . 6 8}[\mathbf{0 . 6 4 , 0 . 7 4 ]}$ \\
Monotherapy (second line) \\
OAK & $0.91[0.77,1.07]$ \\
POPLAR & $0.88[0.66,1.16]$ \\
Subtotal (95\% Cl) & $\mathbf{0 . 9 0}[\mathbf{0 . 7 8 , 1 . 0 4 ]}$ \\
Total (95\% Cl) & $\mathbf{0 . 7 3 [ 0 . 6 8 , 0 . 7 7 ]}$
\end{tabular}

B

\begin{tabular}{|c|c|c|c|}
\hline Study or Subgroup & $\begin{array}{l}\text { Hazard Ratio } \\
\text { IV, Fixed, } 95 \% \mathrm{Cl}\end{array}$ & $\begin{array}{l}\text { Hazarc } \\
\text { IV, Fixed }\end{array}$ & $\begin{array}{l}\text { d Ratio } \\
\text { d, } 95 \% \mathrm{Cl}\end{array}$ \\
\hline \multicolumn{4}{|c|}{ Combination therapy (first line) } \\
\hline IMpassion130 & $0.88[0.72,1.08]$ & & \\
\hline IMpower130 & $0.73[0.57,0.93]$ & - & \\
\hline IMpower133 & $0.64[0.45,0.91]$ & & \\
\hline IMpower150 & $0.83[0.65,1.06]$ & & \\
\hline Subtotal $(95 \% \mathrm{Cl})$ & $0.80[0.70,0.90]$ & & \\
\hline \multicolumn{4}{|c|}{ Monotherapy (second line) } \\
\hline IMvigor211 & $0.83[0.69,1.00]$ & $\rightarrow-$ & \\
\hline OAK & $0.72[0.60,0.88]$ & $\because-$ & \\
\hline POPLAR & $0.68[0.49,0.94]$ & & \\
\hline Subtotal $(95 \% \mathrm{Cl})$ & $0.76[0.67,0.86]$ & & \\
\hline \multirow[t]{3}{*}{ Total $(95 \% \mathrm{Cl})$} & $0.78[0.71,0.85]$ & 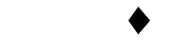 & \\
\hline & & 0.5 & 2 \\
\hline & & $\begin{array}{r}\text { Favors } \\
\text { Atezolizumab }\end{array}$ & $\begin{array}{l}\text { Favors } \\
\text { Standard therapy }\end{array}$ \\
\hline
\end{tabular}

IV, Fixed, $95 \%$

\section{Liver metastasis}

Hazard Ratio Hazard Ratio

IV, Fixed, $95 \% \mathrm{Cl} \quad$ IV, Fixed, $95 \% \mathrm{Cl}$

(IV, Fixed,
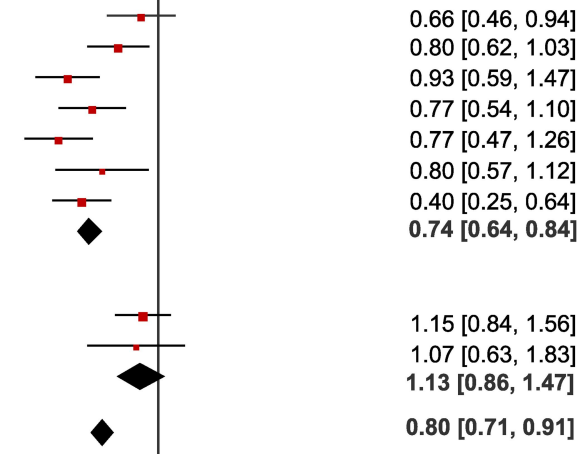

$$
0.5
$$$$
\text { Favors Favors }
$$

Atezolizumab Standard therapy

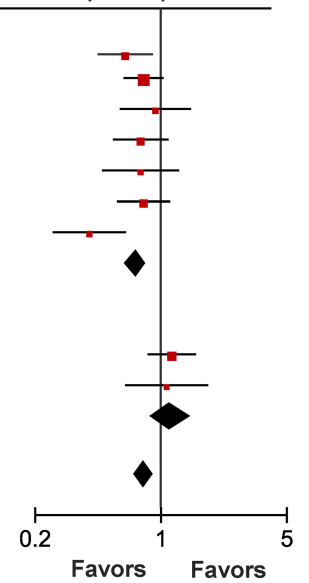

Atezolizumab Standard therapy
Hazard Ratio Hazard Ratio

IV, Fixed, $95 \%$ Cl IV, Fixed, $95 \% \mathrm{CI}$

$0.77[0.58,1.02]$

$1.04[0.63,1.72]$

$0.80[0.57,1.12]$

$0.54[0.34,0.85]$

$0.77[0.64,0.92]$

$0.84[0.64,1.10]$

$0.82[0.59,1.14]$

$0.64[0.37,1.10]$

$0.80[0.66,0.98]$

$0.78[0.69,0.89]$

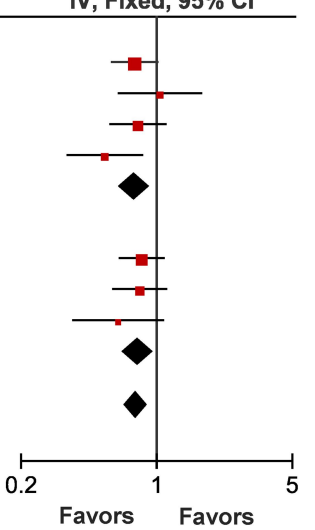

Atezolizumab Standard therapy

C

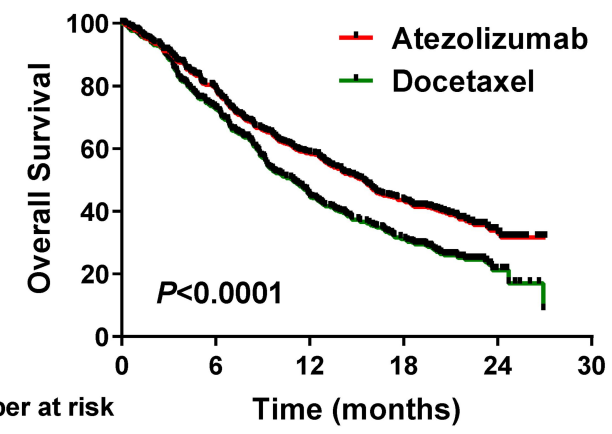

$\begin{array}{lllllll}\text { Atezolizumab } & 453 & 348 & 250 & 181 & 32 & - \\ \text { Docetaxel } & 441 & 289 & 174 & 117 & 20 & \text { - }\end{array}$
D

Liver metastasis

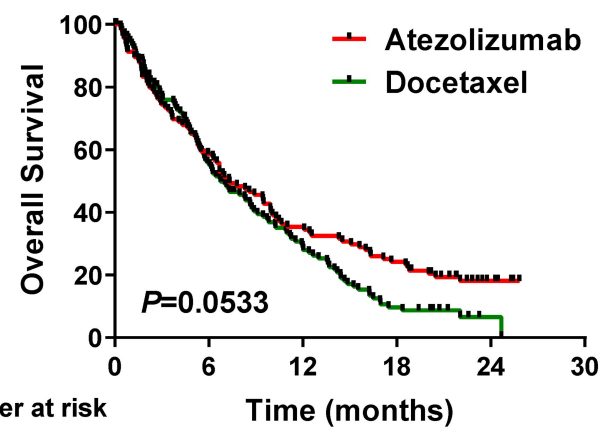

$\begin{array}{lllllll}\text { Atezolizumab } & 116 & 63 & 38 & 26 & 7 & - \\ \text { Docetaxel } & 127 & 66 & 32 & 10 & 1 & \text { - }\end{array}$

Figure I The efficacy of atezolizumab versus standard therapy in terms of liver metastasis (LM). Forest plots of hazard ratios (HRs) with $95 \%$ confidence intervals (Cls) for (A) progression-free survival (PFS) and (B) overall survival (OS) in pan-cancer across treatment lines, comparing atezolizumab and standard therapy in patients with and without LM respectively. Kaplan-Meier estimates of OS with atezolizumab versus docetaxel in (C) LM population and (D) non-LM population in an individual non-small cell lung cancer cohort. 
atezolizumab (mOS 7.33 vs 6.74 months, $P=0.053$ ), the difference between the two treatments did not reach a conventional level of statistical significance (Figure 1D).

\section{Predictive Role of PD-LI in the Liver Metastatic Population Receiving Immunotherapy}

An exploratory analysis of predictive biomarkers was conducted in the liver metastatic population of the OAK cohort, in which 177 (20.8\%) patients harbored LM at baseline. While evaluating the degree of improvement in OS after atezolizumab treatment compared to docetaxel therapy, a strong PD-L1 expression (TC3 or IC3) was also observed, emerging as a prominent biomarker among all candidates, with an HR of 0.395 (95\% CI: 0.167-0.937), among patients with atezolizumabimproved OS (Table 1).

We therefore evaluated the predictive potential of PD-L1 values regarding OS of patients with and without LM in the

Table I Biomarker Screening by Calculating Hazard Ratio of Atezolizumab versus Docetaxel with $95 \%$ Confidence Interval (Cl) and Corresponding P-value for Each Clinical Characteristic in the Liver Metastatic Population from the OAK Cohort

\begin{tabular}{|c|c|c|c|c|c|}
\hline Clinical Characteristic & Number (Doce) & Number (Atezo) & Hazard Ratio (Atezo vs Doce) & $95 \% \mathrm{Cl}$ & $P$-value \\
\hline \multicolumn{6}{|l|}{ PD-LI } \\
\hline $\mathrm{TC} 3$ or IC3 & 13 & 15 & 0.395 & $0.167-0.937$ & 0.015 \\
\hline $\mathrm{TC} 0 / \mathrm{I} / 2$ and $\mathrm{ICO} / \mathrm{I} / 2$ & 80 & 68 & 0.986 & $0.689-1.4 I I$ & 0.939 \\
\hline \multicolumn{6}{|l|}{ bTMB Status } \\
\hline bTMB-High & 19 & 18 & 0.660 & $0.327-1.329$ & 0.216 \\
\hline bTMB-Low & 57 & 47 & 0.762 & $0.498-1.166$ & 0.207 \\
\hline \multicolumn{6}{|l|}{ SLD } \\
\hline$<2$ & 16 & 9 & 0.917 & $0.378-2.228$ & 0.840 \\
\hline$\geq 2$ & 78 & 74 & 0.776 & $0.546-1.103$ & 0.155 \\
\hline \multicolumn{6}{|l|}{ Metastatic Sites } \\
\hline$<4$ & 52 & 38 & 0.929 & $0.584-1.477$ & 0.754 \\
\hline$\geq 4$ & 42 & 45 & 0.707 & $0.442-1.131$ & 0.140 \\
\hline \multicolumn{6}{|l|}{ ECOG PS } \\
\hline 0 & 29 & 26 & 0.700 & $0.379-1.293$ & 0.252 \\
\hline I & 65 & 57 & 0.898 & $0.610-1.323$ & 0.584 \\
\hline \multicolumn{6}{|l|}{ Race } \\
\hline Asian & 20 & 16 & 0.730 & $0.352-1.517$ & 0.394 \\
\hline White & 68 & 62 & 0.823 & $0.565-1.201$ & 0.311 \\
\hline Other & 6 & 5 & 1.416 & $0.322-6.236$ & 0.642 \\
\hline \multicolumn{6}{|l|}{ Gender } \\
\hline Male & 52 & 55 & 0.842 & $0.553-1.283$ & 0.415 \\
\hline Female & 42 & 28 & 0.792 & $0.469-1.335$ & 0.383 \\
\hline \multicolumn{6}{|l|}{ Histology } \\
\hline Squamous & 20 & 24 & 0.561 & $0.292-1.078$ & 0.068 \\
\hline Non-Squamous & 74 & 59 & 0.930 & $0.635-1.364$ & 0.710 \\
\hline \multicolumn{6}{|l|}{ Prior Chemotherapy } \\
\hline I & 72 & 66 & 0.772 & $0.532-1.123$ & 0.174 \\
\hline 2 & 22 & 17 & 1.000 & $0.508-1.968$ & 1.000 \\
\hline \multicolumn{6}{|l|}{ Smoking Status } \\
\hline Never & 22 & 18 & 0.977 & $0.483-1.976$ & 0.948 \\
\hline Previous/Current & 72 & 65 & 0.760 & $0.525-1.102$ & 0.141 \\
\hline
\end{tabular}

Abbreviations: SLD, sum of the longest diameters; Doce, docetaxel; Atezo, atezolizumab; bTMB, tumor mutation burden in blood; ECOG PS, eastern cooperative oncology group performance status. 
OAK cohort. Among the liver metastatic population, Prolonged OS was observed in patients with strong PD-L1 expression after atezolizumab treatment compared with those treated with docetaxel. (mOS 16.33 vs 4.83 months, $P=$ 0.0150) (Figure 2B); however, OS was generally similar between patients with weak or negative PD-L1 expression regardless of received treatment (mOS 6.31 vs 7.03 months, $P=0.9394$ ) (Figure 2C). These last results are responsible for the failure for reaching statistical significance among treatments in the whole liver metastatic population (Figure 2A). In contrast, OS consistently favored atezolizumab over docetaxel in the non-liver metastatic population (mOS 15.54 vs 10.81 months, $P=0.0008$ ) (Figure 2D), both in the PD-L1 strong expression subgroup (mOS 22.18 vs 10.25 months, $P=$ 0.0006) (Figure 2E) and the weak or negative expression subgroup (mOS 14.06 vs 10.81 months, $P=0.0405$ ) (Figure 2F).

\section{Combination of PD-LI and LM for the Prediction of ICB Treatment}

Based on our findings, we speculated that both the information on PD-L1 expression and liver metastatic status might provide guidance in clinical decision-making for patients treated with atezolizumab. As expected, inferior OS was significantly associated with LM in the OAK cohort (mOS 7.33 vs 15.47 months, $P<0.0001$ ) (Figure $3 \mathrm{~A})$. The inclusion of PD-L1 expression status further enhanced the predictive value of response to atezolizumab $(P<0.0001)$ (Figure 3C). Remarkably, the LM/PD-L1 ${ }^{+}$ subgroup demonstrated increased long-term survival prospects compared with the LM/PD-L1 ${ }^{-}$subgroup (mOS 16.33 vs 6.31 months, $P=0.0945$ ) (Figure 3C). The clinical benefit in the $\mathrm{LM} / \mathrm{PD}-\mathrm{L}^{+}{ }^{+}$subgroup was similar to that seen in the non-LM/PD-L1 ${ }^{-}$subgroup (mOS 16.33 vs 14.06 months, $P=0.8730$ ) (Figure $3 \mathrm{C}$ ). Furthermore, the predictive value of the combination of PD-L1 expression and liver metastatic status was confirmed in the IMvigor210 cohort. Similarly, the presence of LM was a negative predictive factor for atezolizumab (mOS 5.62 vs 11.93 months, $P<0.0001$ ) (Figure 3B). Kaplan-Meier survival curves revealed that the LM/PD-L1 ${ }^{+}$subgroup had a significantly greater long-term survival benefit than the LM/PD-L1 ${ }^{-}$subgroup (mOS 9.76 vs 3.61 months, $P=$ 0.0006) (Figure 3D); additionally, no significant difference in OS was observed between the LM/PD-L1 ${ }^{+}$and non-LM subgroups (Figure 3D).
A

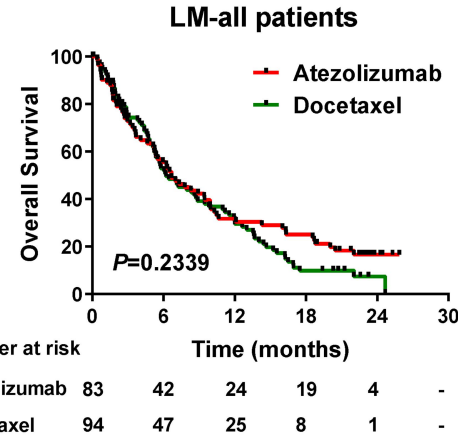

D

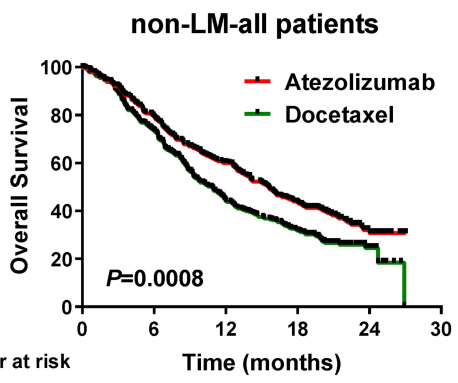

Number at risk

$\begin{array}{llllllll}\text { Atezolizumab } & 342 & 263 & 194 & 138 & 24 & \text { - }\end{array}$

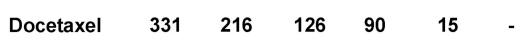
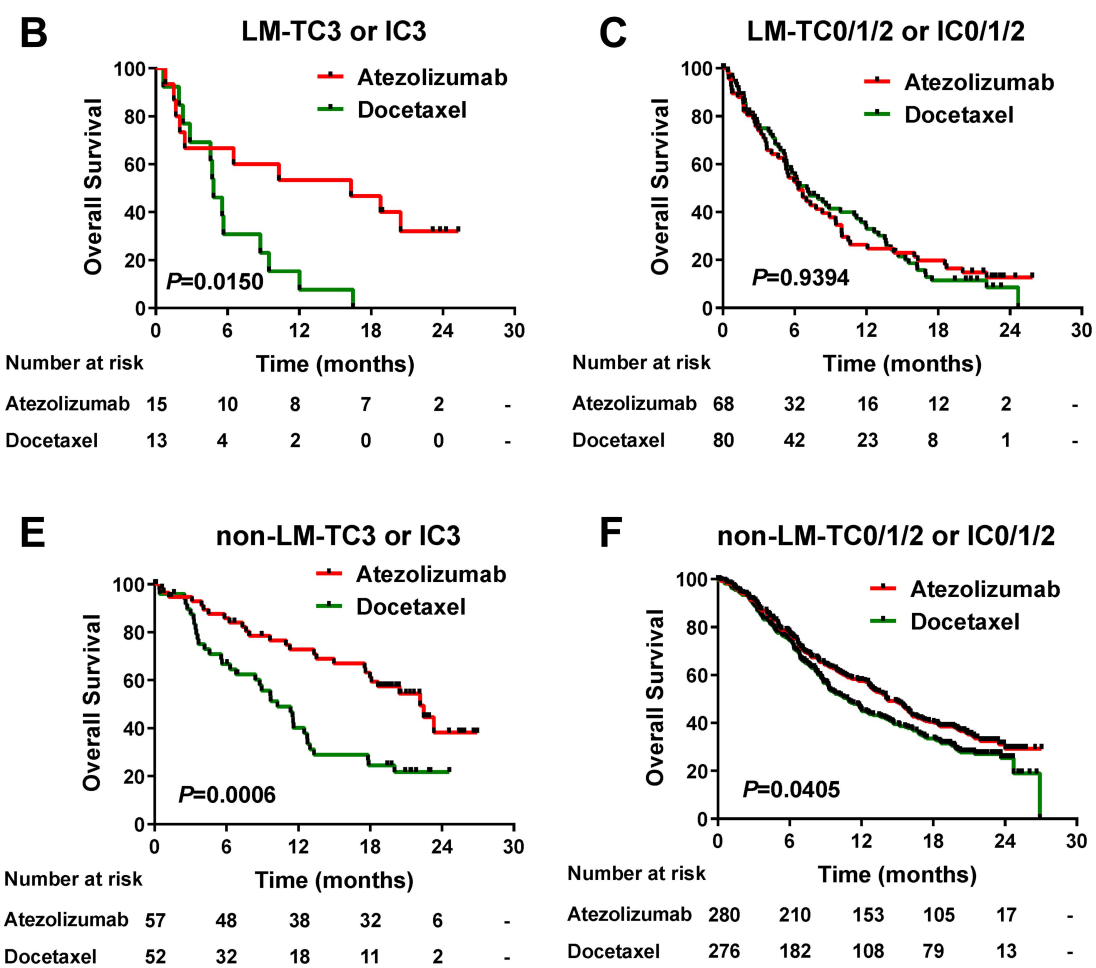

$\mathbf{F}$

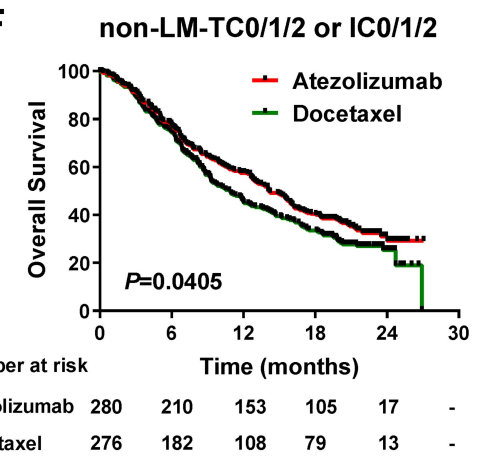

Figure 2 Impact of PD-LI strong expression on efficacy of atezolizumab versus chemotherapy regarding liver metastasis (LM). Kaplan-Meier estimates of overall survival (OS) comparing atezolizumab and docetaxel in (A) all LM patients, (B) LM patients with a PD-LI expression level of TC3 or IC3, (C) LM patients with a PD-LI expression level of TC 0/I/2 or IC0/I/2, (D) all non-LM patients, (E) non-LM patients with TC3 or IC3, and (F) non-LM patients with TC0/I/2 or IC0/I/2. 


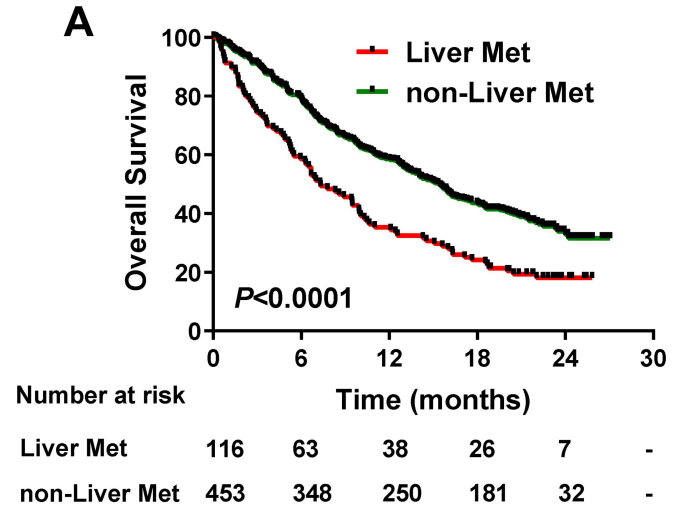

\section{C}

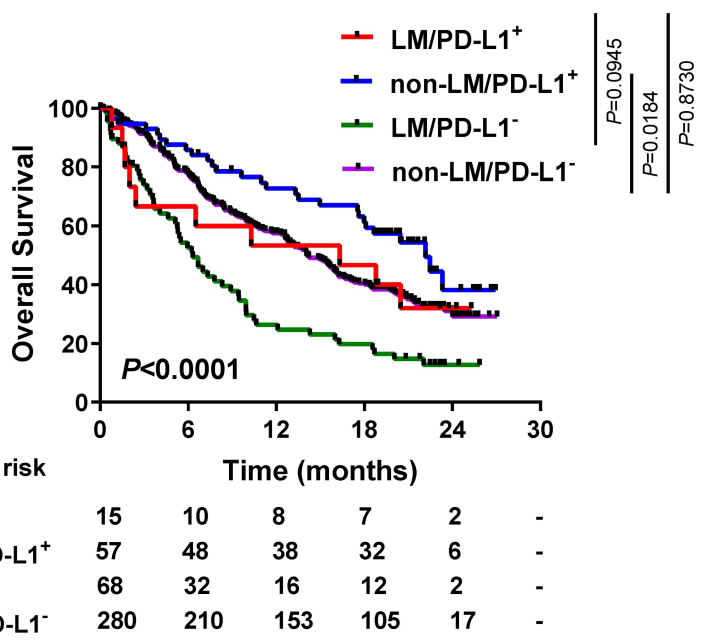

B

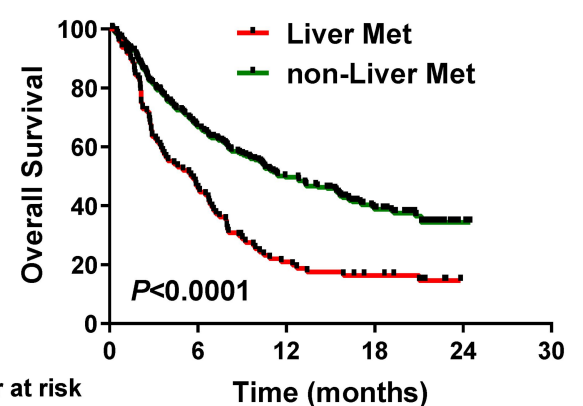

Number at risk

$\begin{array}{lllllll}\text { Liver Met } & 97 & 44 & 19 & 11 & 0 & - \\ \text { non-Liver Met } & 250 & 161 & 119 & 74 & 2 & \text { - }\end{array}$

D

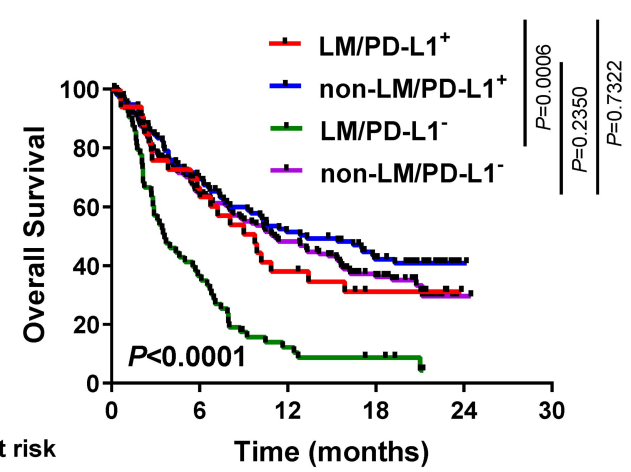

Number at risk

$\begin{array}{lllllll}\text { LM/PD-L1 }^{+} & 33 & 21 & 12 & 7 & 0 & - \\ \text { non-LM/PD-L1 }^{+} & 96 & 66 & 48 & 34 & 1 & - \\ \text { LM/PD-L1 }^{-} & 64 & 23 & 7 & 4 & 0 & - \\ \text { non-LM/PD-L1 }^{-} & 153 & 94 & 70 & 39 & 1 & -\end{array}$

Figure 3 Combination of PD-LI expression and liver metastasis (LM) for risk stratification of patients received atezolizumab. Kaplan-Meier estimates of overall survival (OS) stratified by LM status in (A) the OAK cohort and (B) the IMvigor210 cohort. Kaplan-Meier estimates of OS stratified by LM status and PD-LI expression level in (C) the OAK cohort and (D) the IMvigor210 cohort.

To uncover the underlying mechanism of PD-L1 strong expression in reshaping the immune microenvironment, exploratory translational analyses were performed in the IMvigor210 cohort. Although the liver metastatic population was more likely to exhibit a non-inflamed phenotype (immune excluded and immune desert) than those without liver metastasis (Figure 4A), the occurrence of PD-L1 strong expression significantly reversed the situation, resulting in a larger proportion of immune inflamed phenotype in the LM/PD-L1 ${ }^{+}$subgroup than in the LM/PD$\mathrm{L1}^{-}$and non-LM/PD-L1 ${ }^{-}$subgroups (Figure 4B). Furthermore, transcriptomic analysis revealed that a PDL1 strong expression was associated with a higher level of gene expression in the $\mathrm{T}$ effector cell activation signature (Figure 4C) and higher immune scores of infiltrated immune cells, including $\mathrm{CD}^{+} \mathrm{T}$ cells, NK cells, macrophages, and dendritic cells (Figure S2), which sensitize the LM/PD-L1 ${ }^{+}$subgroup to monoimmunotherapy.

\section{Discussion}

Despite the improvements achieved against advanced-stage cancer by exploiting the cancer-immune set point, the area still calls for much attention on investigations into organspecific immunity. LM is characterized by a poor prognosis and a unique immune microenvironment. However, many problems remain unsolved in this field. Therefore, we investigated the association between LM and clinical outcomes with atezolizumab versus standard of care therapy across different lines of settings, from a pan-cancer standpoint. Conclusions drawn from the pooled analysis were further confirmed with an individual analysis of NSCLC involving trial data from OAK and POPLAR. In contrast with the 
A

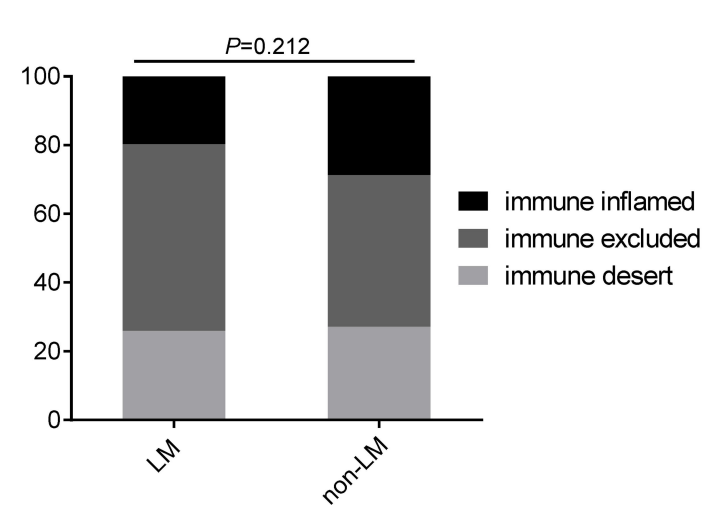

B

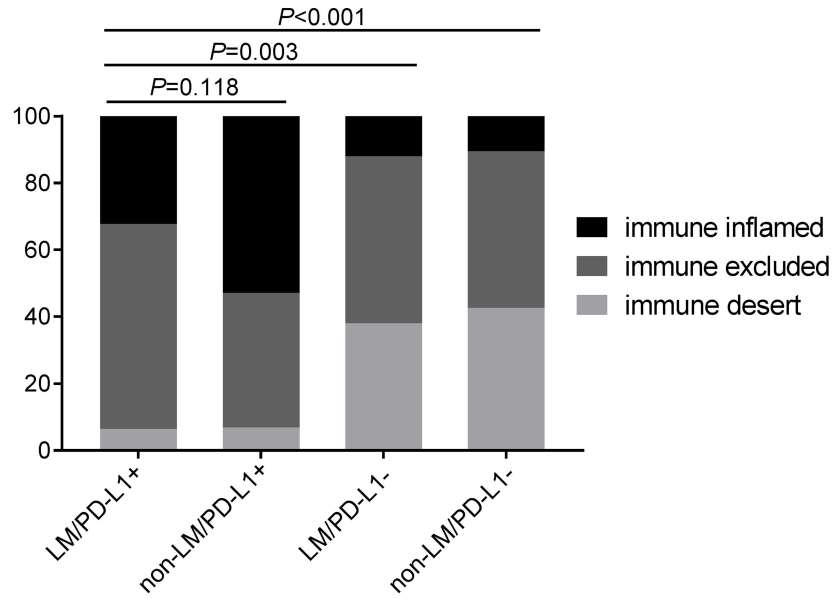

C

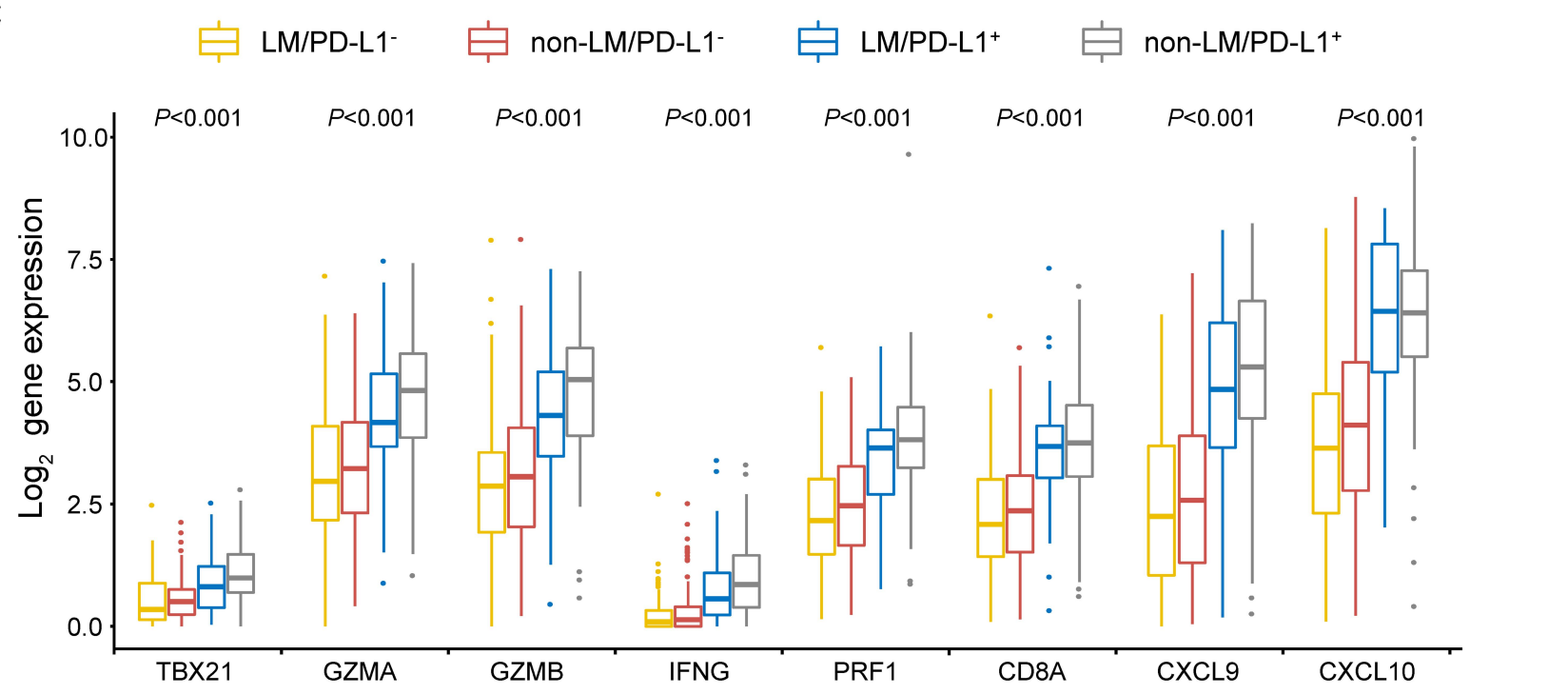

Figure 4 Association of PD-LI strong expression and liver metastasis (LM) with immune profiles. Immune phenotype distributions by (A) liver metastatic status and (B) combination of liver metastatic status and PD-LI strong expression status. (C) T-effector gene expression as a function of PD-LI strong expression and liver metastatic status.

perspectives in most previous studies, we found that there was an OS benefit for patients with LM receiving an atezolizumab treatment compared with those receiving a standard therapy; however, the superiority of the treatment in liver metastatic patients was limited without a population screening in the second-line setting, so we searched for tailored atezolizumab-advantageous subgroups among these patients. Finally, PD-L1 strong expression was found to be a significant biomarker for atezolizumab-advantageous subgroup selection in the liver metastatic population. The underlying evidence that these subgroups of patients harbored an immune-flamed microenvironment were also provided, making their benefits from immunotherapy reasonable.
Until now, controversy remains as to whether patients with LM can benefit from ICB immunotherapy. In the first-line setting, the IMpower150 trial reported prolonged survival with immunotherapy versus standard therapy in patients with $\mathrm{LM}^{24}$ whereas IMpower130 demonstrated similar outcomes between treatments of immunotherapy and standard therapy. ${ }^{25}$ For the second-line setting, immunotherapy was demonstrated to have an OS benefit over standard therapy in CheckMate017 and CheckMate057, ${ }^{11}$ but no significant difference was observed in the POPLAR and OAK studies. ${ }^{19,20}$ However, these investigations into immunotherapy efficacy in LM leave several questions open. Our pooled analysis was designed to provide 
a legible treatment paradigm for patients with LM when receiving immunotherapy across treatment lines in various cancers. It was demonstrated that liver metastatic patients consistently obtained clinical benefits derived from atezolizumab versus standard therapy. However, second-line patients benefited from atezolizumab to a lesser extent than those that received it in a first-line combination therapy. Thus, it might be proposed that patients with LM can be recommended to receive combination immunotherapy in the first-line setting. However, for the second-line regimen, only a subset of patients exhibited durable responses to monotherapy based on our study, who should be selected and screened by biomarkers cautiously.

It is also worth noting that liver-specific immunity should not be underestimated in studies regarding LM, which might be the key to uncover the implications of ICB therapies. The immune response in the liver is characterized by the induction of immune tolerance, ${ }^{26}$ and cancer cells can harness the immunosuppressive microenvironment to survive and strive. ${ }^{5}$ The immune contexture in the liver is also unique in that it contains a high frequency of NK cells and myeloid-derived Kupffer cells in comparison with other tissues. ${ }^{6,13}$ A recent study reported that liver resident NK cells could thwart the function of hepatic T cells via upregulation of PD-L1, ${ }^{13}$ which implies that PD-L1 expression on immunosuppressive cells plays a vital role in the immune tolerance interactions in the liver. Additionally, the liver is an organ with numerous vasculatures, consisting of myeloid-derived suppressor cells (MDSCs) and hepatic stellate cells, which establish barriers for cytotoxic $\mathrm{T}$ cells to infiltrate, get activated, and attack tumor cells. ${ }^{5}$ Based on aforementioned characteristics, the immune microenvironment of patients with LM can be stratified as "TIL(-)PD-L1( \pm )" according to a framework previously proposed; ${ }^{27}$ combination immunotherapy in the first-line setting would therefore be reasonable. Standard chemotherapeutic agents have been reported to induce immunogenic cell death and thus induce a favorable immune microenvironment in the metastasized liver. $^{28,29}$ Furthermore, the liver tissue is highly vascularized, which impedes $\mathrm{T}$ cell infiltration; thus, the combination of antiangiogenic therapy and immunotherapy might benefit patients with $\mathrm{LM}^{24}$ However, as for the secondline monotherapy, the efficacy of immunotherapy was directly affected by the microenvironment orchestrated in the liver. ${ }^{30}$ Heterogeneous subgroups that responded differently to single-agent immunotherapeutics existed. In this regard, it is of great clinical significance to identify subgroups that really benefit from monoimmunotherapy in these patients.

Our results showed that atezolizumab may benefit some patients with LM, comparing to docetaxel in the second-line setting. It is of interest to see the efficacy of atezolizumab combined with docetaxel. Atezolizumab plus taxanes have been reported for advanced triplenegative breast cancer and advanced lung cancer. ${ }^{17,24,25}$ For liver metastasis patients in these clinical study, IMpower 150 study showed that combination therapy significantly prolongs survival compared to standard chemotherapy (mOS 13.3 vs 9.4 months, 95\% CI: 0.33-0.82). ${ }^{24}$ However, IMpassion 130 and IMpower 130 trail suggested that the efficacy of the combination group was comparable to that of the standard treatment group in liver metastasis patients. ${ }^{17,25}$ At present, many different combination regimens for immunotherapy and chemotherapy were available in clinical use. The pooled analysis in our study showed that atezolizumab combination demonstrated an OS benefit compared with standard therapy in the liver metastatic population. Preclinical studies demonstrate that the majority of chemotherapeutic drugs have been shown to exert immunostimulatory effects, either by inhibiting immunosuppressive cells and/or activating effector cells, or by increasing immunogenicity and increasing T-cell infiltration. ${ }^{31-33}$ Further studies should focus on determining the optimal drug combination, sequence effects and optimal concentration-time profiles.

Although the presence of liver metastasis has been categorized into immune suppressive profiles, ${ }^{30}$ there still exists a proportion of patients harboring PD-L1 strong expression that have reversed the non-inflamed phenotype into an inflamed one. These subgroups of patients had a higher percentage of immune-inflamed profiles, and markers representing TIL infiltration and activation were also significantly increased, which consequently improved the clinical benefit of immunotherapy. An alternative cutoff value of PD-L1 was also adopted. We stratified PD-L1 expression status into positive (TC1/2/3 or $\mathrm{IC} 1 / 2 / 3)$ and negative (TC0 and IC0) subgroups, and conducted similar survival analyses, but failed to observe significant differences in PD-L1 positive patients without liver metastasis (Figure S3). Furthermore, we found that the TC1/2 or IC1/ 2 subgroup (weak positive) did not benefit significantly from atezolizumab versus docetaxel irrespective of liver metastatic status (Figure S4), which was consistent with previous studies. $^{34}$ These findings suggest the rationality of PD-L1 strong positive expression level as a biomarker 
for screening patients with LM, especially for those receiving atezolizumab treatment. It is of high clinical value, since we had proposed that patients with LM should not be roughly excluded from receiving monoimmunotherapy. When the metastatic status of liver is used in combination with PD-L1 strong expression, individuals who may respond to atezolizumab treatment can be selected. This tailored and personalized immunotherapy can improve their prognosis and avoid treatment-related side effects, since combination therapies or conventional therapies may add considerable toxicities to patients with LM.

\section{Conclusions}

This comprehensive study systematically evaluated the efficacy of atezolizumab versus conventional therapy in a liver metastatic population across different treatment lines among various tumor types. It was evidenced that patients with LM could benefit from immunotherapy, but advantageous subgroups should be screened for second-line regimens of immunotherapy. The combination signature of PDL1 expression level and liver metastatic status can be proposed as a way to select advantageous responders for immunotherapy, especially for the second-line setting. This study might offer new clues for clinical practices in liver metastatic patients receiving immunotherapy, which provides another step toward filling the gap in this area.

\section{Acknowledgments}

We would like to thank Professor David S. Shames and Dr. Jing-Yu Zhang from Genentech, Inc. for providing individual clinical data of OAK and POPLAR clinical trials.

\section{Funding}

This work was supported by Guangzhou Science and Technology Foundation [No.201803010059] and the National Natural Science Foundation for Young Scientists of China [No.81802863 and 81902353].

\section{Disclosure}

We declare that we have no conflict of interest.

\section{References}

1. Barlesi F, Vansteenkiste J, Spigel D, et al. Avelumab versus docetaxel in patients with platinum-treated advanced non-small-cell lung cancer (JAVELIN Lung 200): an open-label, randomised, Phase 3 study. Lancet Oncol. 2018;19(11):1468-1479. doi:10.1016/S1470-2045(18) 30673-9
2. Balar AV, Galsky MD, Rosenberg JE, et al. Atezolizumab as first-line treatment in cisplatin-ineligible patients with locally advanced and metastatic urothelial carcinoma: a single-arm, multicentre, Phase 2 trial. Lancet. 2017;389(10064):67-76. doi:10.1016/S0140-6736(16) 32455-2

3. Liu L, Bai X, Wang J, et al. Combination of TMB and CNA Stratifies Prognostic and Predictive Responses to Immunotherapy Across Metastatic Cancer. Clin Cancer Res. 2019;25(24):7413-7423. doi:10.1158/1078-0432.CCR-19-0558

4. Dong ZY, Zhong WZ, Zhang XC, et al. Potential Predictive Value of TP53 and KRAS Mutation Status for Response to PD-1 Blockade Immunotherapy in Lung Adenocarcinoma. Clin Cancer Res. 2017;23:3012-3024. doi:10.1158/1078-0432.CCR-16-2554

5. Hegde PS, Chen DS. Top 10 Challenges in Cancer Immunotherapy. Immunity. 2020;52:17-35. doi:10.1016/j.immuni.2019.12.011

6. Salmon H, Remark R, Gnjatic S, et al. Host tissue determinants of tumour immunity. Nat Rev Cancer. 2019;19:215-227. doi:10.1038/ s41568-019-0125-9

7. Jenne CN, Kubes P. Immune surveillance by the liver. Nat Immunol. 2013;14(10):996-1006. doi:10.1038/ni.2691

8. Milette S, Sicklick JK, Lowy AM, et al. Molecular Pathways: targeting the Microenvironment of Liver Metastases. Clin Cancer Res. 2017;23:6390-6399. doi:10.1158/1078-0432.CCR-15-1636

9. Riihimäki M, Hemminki A, Fallah M, et al. Metastatic sites and survival in lung cancer. Lung Cancer. 2014;86(1):78-84. doi:10.1016/j.lungcan.2014.07.020

10. Hoang T, Dahlberg SE, Sandler AB, et al. Prognostic models to predict survival in non-small-cell lung cancer patients treated with first-line paclitaxel and carboplatin with or without bevacizumab. $J \quad$ Thorac Oncol. 2012;7:1361-1368. doi:10.1097/ JTO.0b013e318260e106

11. Vokes EE, Ready N, Felip E, et al. Nivolumab versus docetaxel in previously treated advanced non-small-cell lung cancer (CheckMate 017 and CheckMate 057): 3-year update and outcomes in patients with liver metastases. Ann Oncol. 2018;29(4):959-965. doi:10.1093/ annonc/mdy041

12. McKay RR, Kroeger N, Xie W, et al. Impact of bone and liver metastases on patients with renal cell carcinoma treated with targeted therapy. Eur Urol. 2014;65(3):577-584. doi:10.1016/j. eururo.2013.08.012

13. Zhou J, Peng H, Li K, et al. Liver-Resident NK Cells Control Antiviral Activity of Hepatic T Cells via the PD-1-PD-L1 Axis. Immunity. 2019;50(2):403-417. doi:10.1016/j.immuni.2018.12.024

14. Brodt P. Role of the microenvironment in liver metastasis: from preto prometastatic niches. Clin Cancer Res. 2016;22(24):5971-5982. doi:10.1158/1078-0432.CCR-16-0460

15. Kato K, Cho BC, Takahashi M, et al. Nivolumab versus chemotherapy in patients with advanced oesophageal squamous cell carcinoma refractory or intolerant to previous chemotherapy (ATTRACTION-3): a multicentre, randomised, open-label, phase 3 trial. Lancet Oncol. 2019;20(11):1506-1517. doi:10.1016/S14702045(19)30626-6

16. Rini BI, Powles T, Atkins MB, et al. Atezolizumab plus bevacizumab versus sunitinib in patients with previously untreated metastatic renal cell carcinoma (IMmotion151): a multicentre, open-label, phase 3, randomised controlled trial. Lancet. 2019;393(10189):2404-2415. doi:10.1016/S0140-6736(19)30723-8

17. Schmid P, Rugo HS, Adams S, et al. Atezolizumab plus nab-paclitaxel as first-line treatment for unresectable, locally advanced or metastatic triple-negative breast cancer (IMpassion130): updated efficacy results from a randomised, double-blind, placebo-controlled, phase 3 trial. Lancet Oncol. 2020;21(1):44-59. doi:10.1016/S1470-2045(19)30689-8

18. Crispe IN. Immune tolerance in liver disease. Hepatology. 2014;60 (6):2109-2117. doi:10.1002/hep.27254 
19. Fehrenbacher L, Spira A, Ballinger M, et al. Atezolizumab versus docetaxel for patients with previously treated non-small-cell lung cancer (POPLAR): a multicentre, open-label, phase 2 randomised controlled trial. Lancet. 2016;387(10030):1837-1846. doi:10.1016/ S0140-6736(16)00587-0

20. Rittmeyer A, Barlesi F, Waterkamp D, et al. Atezolizumab versus docetaxel in patients with previously treated non-small-cell lung cancer (OAK): a phase 3, open-label, multicentre randomised controlled trial. Lancet. 2017;389(10066):255-265. doi:10.1016/S01406736(16)32517-X

21. Rosenberg JE, Hoffman-Censits J, Powles T, et al. Atezolizumab in patients with locally advanced and metastatic urothelial carcinoma who have progressed following treatment with platinum-based chemotherapy: a single-arm, multicentre, phase 2 trial. Lancet. 2016;387 (10031):1909-1920. doi:10.1016/S0140-6736(16)00561-4

22. Davoli T, Uno H, Wooten EC, et al. Tumor aneuploidy correlates with markers of immune evasion and with reduced response to immunotherapy. Science. 2017;355(6322):355. doi:10.1126/science. aaf8399

23. Wang J, Sun H, Zeng Q, et al. HPV-positive status associated with inflamed immune microenvironment and improved response to anti-PD-1 therapy in head and neck squamous cell carcinoma. Sci Rep. 2019;9(1):13404. doi:10.1038/s41598-019-49771-0

24. Reck M, Mok TSK, Nishio M, et al. Atezolizumab plus bevacizumab and chemotherapy in non-small-cell lung cancer (IMpower150): key subgroup analyses of patients with EGFR mutations or baseline liver metastases in a randomised, open-label phase 3 trial. Lancet Respir Med. 2019;7:387-401. doi:10.1016/S2213-2600(19)30084-0

25. West H, McCleod M, Hussein M, et al. Atezolizumab in combination with carboplatin plus nab-paclitaxel chemotherapy compared with chemotherapy alone as first-line treatment for metastatic non-squamous non-small-cell lung cancer (IMpower130): a multicentre, randomised, open-label, phase 3 trial. Lancet Oncol. 2019;20:924-937. doi:10.1016/S1470-2045(19)30167-6
26. Crispe IN. The liver as a lymphoid organ. Annu Rev Immunol. 2009;27(1):147-163. doi:10.1146/annurev.immunol.021908.132629

27. Teng MW, Ngiow SF, Ribas A, et al. Classifying Cancers Based on T-cell Infiltration and PD-L1. Cancer Res. 2015;75:2139-2145. doi:10.1158/0008-5472.CAN-15-0255

28. Gadgeel S, Rodríguez-Abreu D, Speranza G, et al. Updated Analysis From KEYNOTE-189: pembrolizumab or Placebo Plus Pemetrexed and Platinum for Previously Untreated Metastatic Nonsquamous Non-Small-Cell Lung Cancer. J Clin Oncol. 2020;38:1505-1517. doi:10.1200/JCO.19.03136

29. Schaer DA, Geeganage S, Amaladas N, et al. The Folate Pathway Inhibitor Pemetrexed Pleiotropically Enhances Effects of Cancer Immunotherapy. Clin Cancer Res. 2019;25:7175-7188. doi:10.1158/ 1078-0432.CCR-19-0433

30. Tumeh PC, Hellmann MD, Hamid O, et al. Liver Metastasis and Treatment Outcome with Anti-PD-1 Monoclonal Antibody in Patients with Melanoma and NSCLC. Cancer Immunol Res. 2017;5 (5):417-424. doi:10.1158/2326-6066.CIR-16-0325

31. Kodumudi KN, Woan K, Gilvary DL, et al. A novel chemoimmunomodulating property of docetaxel: suppression of myeloid-derived suppressor cells in tumor bearers. Clin Cancer Res. 2010;16:4583-4594. doi:10.1158/1078-0432.CCR-10-0733

32. Demaria S, Volm MD, Shapiro RL, et al. Development of tumor-infiltrating lymphocytes in breast cancer after neoadjuvant paclitaxel chemotherapy. Clin Cancer Res. 2001;7:3025-3030.

33. Jackaman C, Majewski D, Fox SA, et al. Chemotherapy broadens the range of tumor antigens seen by cytotoxic $\mathrm{CD} 8(+) \mathrm{T}$ cells in vivo. Cancer Immunol Immunother. 2012;61:2343-2356. doi:10.1007/ s00262-012-1307-4

34. Papadimitrakopoulou V, Cobo M, Bordoni R, et al. IMpower132: PFS and Safety Results with 1L Atezolizumab plus Carboplatin/ Cisplatin plus Pemetrexed in Stage IV Non-Squamous NSCLC. J Thorac Oncol. 2018;13:S332-S333. doi:10.1016/j.jtho.2018.08.262
Cancer Management and Research

\section{Publish your work in this journal}

Cancer Management and Research is an international, peer-reviewed open access journal focusing on cancer research and the optimal use of preventative and integrated treatment interventions to achieve improved outcomes, enhanced survival and quality of life for the cancer patient.

\section{Dovepress}

The manuscript management system is completely online and includes a very quick and fair peer-review system, which is all easy to use. Visit http://www.dovepress.com/testimonials.php to read real quotes from published authors. 